\title{
Zika Virus: The Current Threat
}

\author{
${ }^{1}$ Ruchika Garg, ${ }^{2}$ Richa Singh, ${ }^{3}$ Priya Mahajan, ${ }^{4}$ Soniya Dhiman
}

\begin{abstract}
Mosquito-borne infections and viral outbreaks have bewildered physicians and population at large from time to time. Zika virus and its vector Aedes aegypti are currently bothering the world population, and this infection has affected pregnant women causing microcephaly in their newborns and also has caused Guillain-Barré syndrome (GBS)-like manifestations in affected individuals. Currently, the outbreak is concentrated in the countries of South American continent, but the omnipresence of its vector has made the world community cautious about the potential of its spread; thus the great emphasis is on prevention and vector control strategies to counter Zika virus attack. Consequently, Ministry of Health, Government of India has also taken cognizance of this and issued guidelines to tackle this problem.
\end{abstract}

Keywords: Guillain-Barré syndrome, Mosquito-borne infections, Zika virus.

How to cite this article: Garg R, Singh R, Mahajan P, Dhiman S. Zika Virus: The Current Threat. J South Asian Feder Obst Gynae 2016;8(3):217-221.

Source of support: Nil

Conflict of interest: None

Date of received: 15 April 2016

Date of acceptance: 11 June 2016

Date of publication: July 2016

\section{INTRODUCTION}

Zika virus disease (Zika) is a disease caused by the Zika virus, which is spread to people primarily through the bite of an infected Aedes species mosquito. The most common symptoms of Zika are fever, rash, joint pain, and conjunctivitis (red eyes). The illness is usually mild with symptoms lasting for several days to a week after being bitten by an infected mosquito. People usually do not get sick enough to go to the hospital, and they very rarely die of Zika. For this reason, many people might not realize that they have been infected. However, Zika virus infection during pregnancy can cause a serious birth defect called microcephaly, as well as other severe

\footnotetext{
${ }^{1}$ Assistant Professor, ${ }^{2}$ Professor, ${ }^{3,4}$ Senior Resident

${ }^{1-4}$ Department of Obstetrics and Gynecology, Sarojini Naidu Medical College, Agra, Uttar Pradesh, India

Corresponding Author: Ruchika Garg, Assistant Professor Department of Obstetrics and Gynecology, Sarojini Naidu Medical College, Agra, Uttar Pradesh, India, e-mail: ruchikagargagra@ gmail.com
}

fetal brain defects. Once a person has been infected, he or she is likely to be protected from future infections. It is essential to clarify the doubts and confusion in the minds of physicians and people at large. This article intends to achieve the same with the best known information on Zika till date.

\section{EPIDEMIOLOGY}

Zika virus was first discovered in 1947 in the Zika forest of Uganda in rhesus monkeys. (1) It was later identified in humans in 1968 for the first time in Nigeria. There were only about 14 or 15 cases documented until 2007. In 2007 the first big Zika outbreak was reported in the Yap Island in Micronesia. (2) Other Pacific islands - Fiji, Vanuatu have had periodic outbreaks since then. In May 2015, the Pan American Health Organization (PAHO) issued an alert regarding the first confirmed Zika virus infections in Brazil. The current outbreak has now spread to other countries in South America, Central America, and the Caribbean continents. World Health Organization (WHO) has reported 23 countries and territories in Americas from where local transmission of Zika virus has been reported.

Brazilian researchers have suggested that Zika virus arrived in Brazil from French Polynesia during the 2014 FIFA World Cup tournament (Fig. 1).

Maximum number of cases has been reported till date from Brazil, Colombia, Venezuela, Honduras, and

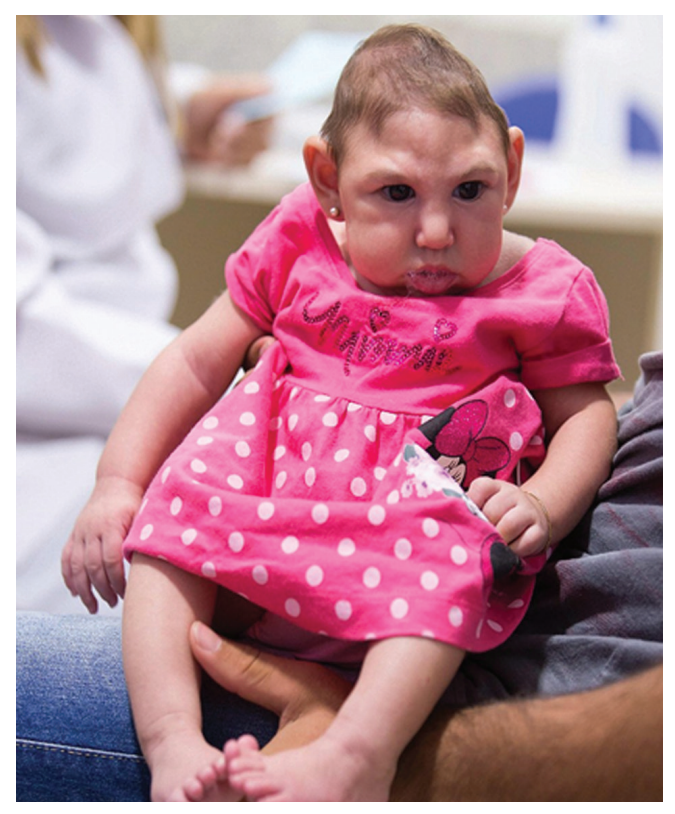




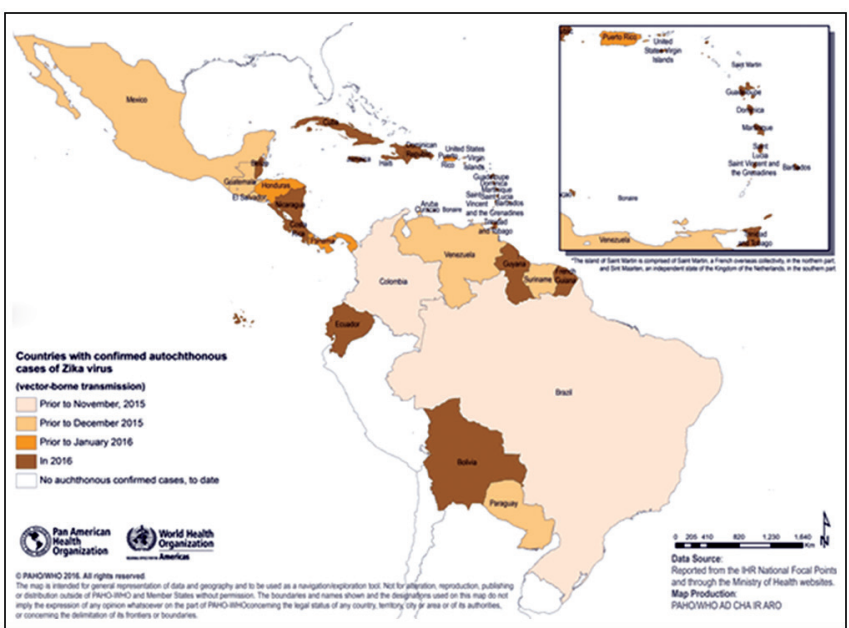

Fig. 1: Countries and territories in the Americas with confirmed autochthonous (vector-borne) Zika virus cases, 2015-2016 (as of 21 April, 2016)

El Salvador. The current outbreak has affected almost 1.3 million people in the world, with the majority of them in Brazil, which is battling with its worst outbreak of Zika. There have been around 35 cases of travel-acquired Zika infections reported from United States. On 1 February, 2016 WHO declared Zika to be a Public Health Emergency of International Concern (PHEIC).

In the epidemiological update on 5 February, 2016 of European Centre for Diseases Prevention and Control, 36 countries or territories have reported autochthonous cases of Zika virus infection in the past 9 months. These countries are all of South American continent. In Brazil, the most affected country, the latest data regarding Zika virus according to the Ministry of Health weekly epidemiological update on the monitoring of microcephaly published on 2 February, 2015, suggest that 4,783 suspected cases of microcephaly have been reported since week 43 , of which 1,132 (24\%) were investigated and classified. Of these cases, $404(36 \%)$ have confirmed microcephaly and/or central nervous system (CNS) malformations, and 17/404 (4\%) were positive for Zika virus infection.

Of 76 suspected deaths from microcephaly and congenital CNS malformations, 15 were investigated and confirmed to have microcephaly and/or CNS malformations. Zika virus was detected in tissues from five of them.

According to the latest update (26 April, 2016), there has been a drop in Zika virus cases in Brazil. No precise numbers for this drop are released but the Brazilian government has admitted that Zika has been reported in all its states, totaling more than 1.5 million cases, and more than 4,000 suspected cases of microcephaly in babies.

Currently, till our article went on for printing, there have been no cases of Zika reported from India or the subcontinent.

\section{VIROLOGY AND PATHOGENESIS}

Zika virus is a virus of flavivirus category of arbovirus type. These are RNA viruses which are enveloped viruses and are transmitted by arthropod bites. Zika virus falls into the same category of virus as of the virus causing Dengue, Chikungunya, West Nile Fever, and Yellow fever.

Zika virus has a single-stranded nonsegmented positive sense RNA genome. Arthropod vectors feed themselves on viremic hosts. Arthropods maintain the viruses via transovarian transmission.

Zika virus is primarily transmitted by the bite of Aedes aegypti mosquito from infected patients to others. Zika virus can also be transmitted from pregnant patients to the fetus intrapartum and during time of delivery.

Some observational evidence from a couple of small studies have reported that infection with Zika can pass on the virus to others through sexual intercourse as the virus has been found in the semen of the patients and can also be transmitted through blood. But to further clarify there have been no reports of transmission of Zika through breast milk currently.

Aedes aegypti which is the vector for this virus has a wide presence throughout the world. Zika virus disease thus has the potential for further international spread given the wide geographical distribution of the mosquito vector. Hence, countries like India need to be vigilant.

The incubation period of Zika virus has been reported to be around 7 to 10 days.

The hosts of the virus are primarily monkeys and humans.

The pathogenesis of the virus is hypothesized to start with an infection of dendritic cells near the site of inoculation, followed by a spread to lymph nodes and the bloodstream. Flavivirus generally replicate in the cytoplasm, but Zika virus antigens have been found in infected cell nuclei.

Infection with the virus appears to be linked to the development of unusually small heads and brain damage in newborns (microcephaly).

The most dangerous time is thought to be during the first trimester of pregnancy when some women do not realize they are pregnant.

\section{CLINICAL MANIFESTATIONS}

Zika virus infection leads to fever, rash, joint pain, and conjunctivitis (Table 1). In most cases, infection usually leads to mild symptoms. Zika virus disease typically produces mild and self-limiting symptoms. The proportion of asymptomatic infections may be as high as $80 \%$. Many people might not even realize that they have been infected. Symptoms of infection usually appear 2 to 7 days after being bitten by an infected mosquito. The 
Table 1: Clinical characteristic of sexually transmitted Zika virus in the region of the Americas (as of 21 April 2016)

\begin{tabular}{lll}
\hline Signs and symptoms & Number of cases* & Percentage \\
\hline Rash & 6 & 86 \\
Conjunctivitis & 5 & 71 \\
Fever & 5 & 71 \\
Athralgia & 5 & 71 \\
Myalgia & 4 & 57 \\
Pruritus & 3 & 43 \\
Headache & 2 & 29 \\
\hline
\end{tabular}

clinical features of Zika virus infection may mimic the features of mild dengue fever.

Microcephaly in the newborns have been reported in patients being infected with Zika virus infection. This has been the major cause of concern in Brazil where maximum number such cases have been reported. Some other congenital CNS abnormalities with adverse fetal outcomes and fetal deaths have also been reported.

Brazilian researchers have studied the amniotic fluid in pregnant women diagnosed to have microcephaly in the fetuses. They found Zika virus in it. In January, scientists at the CDC found Zika virus in the brains of two babies with microcephaly, who died within 24 hours of being born. They also found evidence of Zika infection in two pregnancies that ended in miscarriage.

There have also been reports of a sporadic increase in cases of Guillain-Barré Syndrome (GBS) in areas affected with Zika virus infection, thus pointing toward its occurrence due to Zika virus. $62 \%$ cases of GBS in Brazil in recent times have shown symptoms of Zika virus infection prior to neurological symptoms.

\section{DIAGNOSTIC TESTS}

The patients who develop symptoms consistent with Zika virus infection and have history of travel to Zika affected areas in last 2 weeks should report to the health care facilities where they would be tested to confirm the diagnosis.

Zika virus can be tested in following samples: Blood, saliva, and urine. Mostly blood tests are performed. Following tests can be used for detection:

- Polymerase chain reaction (PCR) test: It is useful in the first 3 to 5 days after the onset of symptoms. It helps in the direct detection of Zika virus RNA or specific viral antigens in clinical specimens.

- Serology test: It detects the presence of antibodies but are useful only after 5 days.

The clinicians should keep a high degree of suspicion of Zika infection in those people who present with its symptoms and have history of travel in the areas which have reported to be affected with Zika virus in the last 2 weeks.
As per the directives of Health Ministry of India, NCDC, Delhi and National Institute of Virology (NIV), Pune, have the capacity to provide laboratory diagnosis of Zika in acute febrile stage.

These two institutions would be the apex laboratories to support the outbreak investigation and for confirmation of laboratory diagnosis. Ten additional laboratories would be strengthened by ICMR to expand the scope of laboratory diagnosis. RT-PCR test would remain the standard test. As of now there is no commercially available test for Zika. Serological tests are not recommended by them for the detection. ${ }^{10}$

\section{MANAGEMENT}

Currently, there are no drugs or vaccines available for Zika virus infection.

The management of affected individuals is symptomatic in nature, and following things should be undertaken:

- Adequate fluid intake

- Rest

- Antipyretics in the form paracetamol to treat fever, avoiding aspirin and NSAIDS

- Preventing mosquito bites during infected stage to prevent spread of the disease

- If the patient is pregnant extra care has to be taken to monitor for birth defects and adverse pregnancy outcomes, and termination of pregnancy should be considered in case of documented birth defects during pregnancy.

\section{PREVENTION STRATEGIES}

Companies and scientists are racing to develop a safe and effective vaccine for Zika, but the World Health Organization (WHO) had said it would take at least 18 months to start large-scale clinical trials of potential preventative shots. There are reports of an Indian Biotech company which is ready with two candidate vaccines for ZIKV infection to be tested in preclinical stages.

Hence, prevention of this disease becomes the most crucial method to control the outbreak and its spread.

People should take care to prevent themselves from getting infected with ZIKV which could be undertaken through following routes:

- Preventing mosquito bites by (a) staying indoors, wearing long sleeved shirts and long pants. (b) Using environmental protection agency registered insect repellants. (c) Use mosquito nets. ${ }^{11}$

- Avoiding nonessential travel to countries currently facing the wrath of ZIKV infection.

- Pregnant women should specifically avoid traveling to affected countries. 
- Women of reproductive age residing in affected countries have been advised to avoid getting pregnant currently due to surge of microcephaly cases.

- Travel precautions which should be followed that include travelers to affected areas should take extra care to avoid mosquito bites by personal protective measures. Travelers should monitor themselves after return and in case of development of any symptoms suggestive of ZIKV infection should immediately report to health care facilities.

- Cases of sexual transmission have also been reported, prompting health officials to advise use of condoms, or abstaining from sex, to prevent infection between partners.

- Vector control by vector surveillance, environmental manipulation and modification, biological and chemical control and use of personal protective measures have to be strengthened.

- Information dissemination to the people by various methods like, e.g., use of billboards at airports, hospitals, educating health care professionals and population at large are essential to create awareness about ZIKV and remove panic.

- Surveillance at various stages is very essential to prevent the spread of this outbreak and also to control the infection. Thus pediatricians, obstetricians, and physicians should be sensitized toward monitoring cases and keeping a tab on numbers. In cases of GBS, atypical unexplained neurological manifestations, newborns with birth defects, and microcephaly, the health care providers should keep a high degree of suspicion and elicit histories of travel and report accordingly.

An international and national collaboration at various levels is equally essential element to prevent the control of this outbreak.

\section{WHO RESPONSE}

- World Health Organization is supporting countries to control Zika by taking actions outlined in the "Zika Strategic Response Framework."

- Define and prioritize research into Zika by convening experts and partners.

- Enhance surveillance of Zika virus and potential complications.

- Strengthen capacity in risk communication to help countries meet their commitments under the International Health Regulations.

- Provide training on clinical management, diagnosis, and vector control, including through a number of WHO Collaborating Centers.

- Strengthen the capacity of laboratories to detect the virus.
- Support health authorities to implement vector control strategies aimed at reducing Aedes mosquito populations, such as providing larvicide to treat still water sites that cannot be treated in other ways, such as cleaning, emptying, and covering them.

- Prepare recommendations for clinical care and followup of people with Zika virus, in collaboration with experts and other health agencies.

\section{CONCLUSION}

The increase in number of cases in Zika virus infection and its association with microcephaly and GBS, as reported currently, should not create a panic among the population at large. Sensitization and spread of correct information to all stakeholders including physicians, obstetricians, and pediatricians is very essential. Such virus outbreaks have become very common in recent times, and people have blamed environmental tampering and enhanced international travel for the same. Hence a systemic approach in such cases at various levels needs to be formulated by regulatory and executive authorities. This would prevent panic among the population and would also lead to better handling in future of any such threats.

People planning their summer vacations and holidays to coincide with the upcoming Olympics in Rio De Janeiro should be cautious. The host city has already launched massive drives to target mosquito breeding sites to curb the spread of Zika virus and thus ensure that athletes and visitors remain safe. According to Samdani, with the vector already being present in India, we may not be far from treating a case that could lead to widespread transmission.

\section{BIBLIOGRAPHY}

1. Dick GW, Kitchen SF, Haddow AJ. Zika virus. I. Isolations and serological specificity. Trans R Soc Trop Med Hyg 1952 Sep;46(5):509-520.

2. Duffy MR, Chen TH, Hancock WT, Powers AM, Kool JL, Lanciotti RS, Pretrick M, Marfel M, Holzbauer S, Dubray C, et al. Zika virus outbreak on Yap Island, Federated States of Micronesia. N Engl J Med 2009 Jun 11;360(24):2536-2543.

3. Neurological syndrome, congenital malformations, and Zika virus infection. Implications for public health in the Americas - Epidemiological Alert; 2015 Dec 1.

4. WHO. Zika virus. Fact sheet; 2016 [updated 2016 Jan]. WHO Media Centre [accessed 2016 Jan 30].

5. Romero S. Tears and bewilderment in Brazilian city facing Zika crisis. The New York Times; 2016 Jan 29 [retrieved 2016 Feb 1].

6. European Centre for Disease Prevention and Control. Epidemiological update: outbreaks of Zika virus and complications potentially linked to the Zika virus infection [2016 Feb 5]. ECDC; 2016. 
7. Faye $\mathrm{O}$, Freire CC, Iamarino A, Faye O, de Oliveira JV, Diallo M, Zanotto PM, Sall AA. Molecular evolution of Zika virus during its emergence in the 20th century. PLoS Negl Trop Dis 2014 Jan 9;8(1):e2636.

8. European Centre for Disease Prevention and Control. Zika virus epidemic in the Americas: potential association with microcephaly and Guillain-Barre syndrome (first update) [2016 Jan 21]. Stockholm: ECDC; 2016.
9. Emerging Infectious Diseases, No. 2, 2012 Feb. Available from: www.cdc.gov/eid,vol18.

10. Health Ministry Issues Guidelines on Zika Virus Disease. Press Information Bureau, Government of India, Ministry of Health and Family Welfare; 2016 Feb 2, 16:25 IST.

11. Marcondes CB, Ximenes Mde F. Zika virus in Brazil and the danger of infestation by Aedes mosquitoes. Rev Soc Bras Med Trop 2016 Feb;49(1):4-10. 